\title{
Functional neuroanatomy of three-term relational reasoning
}

\author{
Vinod Goel ${ }^{\mathrm{a}, \mathrm{b}, *}$, Raymond J. Dolan ${ }^{\mathrm{a}, \mathrm{c}}$ \\ ${ }^{a}$ Wellcome Department of Cognitive Neurology, Institute of Neurology, Queens Square, London WC1N 3BG, UK \\ ${ }^{\mathrm{b}}$ Department Of Psychology, York University, Toronto, Ont., Canada M3J 1P3 \\ ${ }^{c}$ Royal Free Hospital School Of Medicine, Roland Hill Street, London NW3, UK
}

Received 16 August 2000; received in revised form 3 January 2001; accepted 2 February 2001

\begin{abstract}
In a recent study we demonstrated that reasoning with categorical syllogisms engages two dissociable mechanisms. Reasoning involving concrete sentences engaged a left hemisphere linguistic system while formally identical arguments, involving abstract sentences, recruited a parietal spatial network. The involvement of a parietal visuo-spatial system in abstract syllogism reasoning raised the question whether argument forms involving explicit spatial relations (or relations that can be easily mapped onto spatial relations) are sufficient to engage the parietal system? We addressed this question in an event-related fMRI study of three-term relational reasoning, using sentences with concrete and abstract content. Our findings indicate that both concrete and abstract three-term relational arguments activate a similar bilateral occipital-parietal-frontal network. However, the abstract reasoning condition engendered greater parietal activation than the concrete reasoning condition. We conclude that arguments involving relations that can be easily mapped onto explicit spatial relations engage a visuo-spatial system, irrespective of concrete or abstract content. (C) 2001 Elsevier Science Ltd. All rights reserved.
\end{abstract}

Keywords: Deductive reasoning; Neuroimaging; fMRI; Spatial reasoning; Mental models; Mental logic; Higher cognitive functions

\section{Introduction}

An important question that concerns cognitive models of reasoning is whether logical reasoning is inherently sentential or spatial. Sentential (mental logic) theories of reasoning claim that deductive reasoning is a rule governed syntactic process [20] where internal representations preserve structural properties of linguistic strings in which the premises are stated. This linguistic hypothesis predicts that language processing mechanisms mediate human reasoning processes. Mental model theories claim that deductive reasoning is a process requiring spatial manipulation and search where internal representations preserve the structural properties of the world (e.g. spatial relations) that the sentences are about. The spatial hypothesis suggests that the neural structures for visuo-spatial processing contribute the basic representational building-blocks used for logical reasoning [11].

* Corresponding author. Tel.: + 1-416-7365121; fax: + 1-4167365814.

E-mail address: vgoel@yorku.ca (V. Goel).
In a recent fMRI study [7] we demonstrated that both linguistic and spatial processing mechanisms are engaged in syllogistic reasoning processes, but under different circumstances. Reasoning involving concrete syllogisms (e.g. 'all dogs are pets; all poodles are dogs $\therefore$ all poodles are pets') engages a left hemisphere temporal linguistic system, while formally identical reasoning tasks involving abstract syllogisms (e.g. 'all $\mathrm{P}$ are $\mathrm{B}$; all $\mathrm{C}$ are $\mathrm{P} \therefore$ all $\mathrm{C}$ are $\mathrm{B}$ ') recruit a parietal spatial network.

The involvement of a parietal visual-spatial system in the abstract syllogism condition, raises the question whether argument forms involving three-term relational items (e.g. 'the apples are in the barrel; the barrel is in the barn; the apples are in the barn' and 'apples are more expensive than pears; pears are more expensive than oranges; apples are more expensive than oranges') are sufficient to engage the parietal system? One rationale for thinking this might be the case is subjects' reported phenomenological experience of using a visuo-spatial strategy during these tasks. Secondly, neuroimaging studies have shown the involvement of the parietal system in the encoding of relational spatial 
information $[15,16]$. To resolve these issues we carried out a single-event, fMRI study of three-term spatial and nonspatial relational arguments with sampling of the BOLD signal during the reasoning component of the task. Our findings indicate that in three-term relational arguments both the concrete and abstract arguments activate a similar occipital-parietal-frontal network.

\section{Method}

\subsection{Subjects}

Fourteen right-handed normal subjects (six males and eight females), with a mean age of 28.57 years $($ S.D. $=4.6)$ and mean education level of 16.78 years $($ S.D. $=2.15)$, volunteered to participate in the study. All subjects gave informed consent and the study was approved by the Joint National Hospital for Neurology and Neurosurgery/Institute of Neurology Ethics Committee.

\subsection{Stimuli}

Sixty contentful and sixty abstract three-term relational arguments were generated. Half the items involved explicit spatial relations such as 'Larry is above Michael', while the other half involved nonspatial relations such as 'Larry is heavier than Michael', that are naturally mapped onto spatial relations. These arguments were evenly divided into the following four forms: determinate consistent (e.g. A is ahead of B; C is ahead of $\mathrm{A} \therefore \mathrm{C}$ is ahead of $\mathrm{B}$ ), determinate inconsistent (e.g. $\mathrm{A}$ is ahead of $\mathrm{B}$; $\mathrm{C}$ is ahead of $\mathrm{A} \therefore \mathrm{C}$ is behind $\mathrm{B}$ ), indeterminate consistent (e.g. $\mathrm{A}$ is in front of $\mathrm{B}$; $\mathrm{A}$ is in front of $\mathrm{C} \therefore \mathrm{B}$ is in front of $\mathrm{C}$ ), and indeterminate inconsistent (e.g. A is above $\mathrm{B}$; $\mathrm{C}$ is below A $\therefore$ C is above B). Half of the arguments were valid, the other half invalid. The concrete arguments contained sentences like 'Larry is standing behind Kirk', while the abstract sentences were of the form ' $\mathrm{L}$ is standing behind K'. All sentences were grammatical and meaningful. The baseline trials consisted of three sentences that did not constitute an argument, by virtue of the fact that the third sentence was unrelated to the first two sentences, for example, 'A is ahead of $\mathrm{B}$; $\mathrm{C}$ is ahead of $\mathrm{A} \therefore \mathrm{F}$ is above $\mathrm{X}$ ' or 'Adam is ahead of Bob; Carol is ahead of Adam $\therefore$ George is next to Mark'. These baseline items were generated by switching around the conclusions of the arguments, thus circumventing the need to introduce new sentences in the baseline. There were thirty items in each of the content and abstract baseline conditions. Examples of each category of stimuli appear in Fig. 1a.

\subsubsection{Stimuli presentation}

Stimuli from all conditions were presented randomly in an event-related design (see Fig. 1b). The beginning of a trial was signaled by an '*'. The sentences appeared on the screen one at a time with the first sentence appearing at $500 \mathrm{~ms}$, the second at $3500 \mathrm{~ms}$, and the last sentence at $6500 \mathrm{~ms}$. All sentences remained on the screen until the end of the trial. The length of trials varied from $10.25-14.35 \mathrm{~s}$, leaving subjects $3.75-7.85 \mathrm{~s}$ (after the presentation of the third sentence) to respond.

\subsubsection{Task}

The task in all trials was the same. Subjects were required to determine whether the given conclusion followed logically from the premises (i.e. whether the argument was valid). In baseline trials, where the first two sentences were related, subjects would need to begin to integrate the premises and construct a representation of the problem (task difficulty and time limitations do not allow subjects the option of waiting until the presentation of the third sentence before deciding to begin integration of the first two sentences), but when the third, unrelated, sentence appeared they could immediately disengage the task and respond 'no'. (This does mean that there is an imbalance between 'yes' and 'no' responses. However, the behavioural data suggests that subjects are not getting locked into a mental set. We have explored a number of baselines and feel the advantages of this one, outweigh the disadvantages.) In trials where the three sentences constituted an argument, subjects would need to continue with the reasoning component of the task after the presentation of the third sentence (reasoning condition). The difference between completing the reasoning task and disengaging after the presentation of the third sentence isolates the reasoning components of interest. This design, involving a time-locked single-event design and an (unblocked) random presentation of trials, circumvents the question about what constitutes an appropriate baseline for reasoning tasks and allows us to keep task instructions constant across all conditions.

Subjects responded by pressing a button on a keypad after the appearance of the last sentence. Subjects were instructed to respond as quickly as possible and move to the next trial if the stimuli advanced before they could respond. Subjects reviewed example stimuli from each condition prior to being scanned to ensure that they understood the task.

\section{3. fMRI scanning technique}

A $2 \mathrm{~T}$ Siemens VISION system (Siemens, Erlangen, Germany) was used to acquire T1 anatomical volume images $(1 \times 1 \times 1.5 \mathrm{~mm}$ voxels $)$ and $48 \mathrm{~T} 2 *$-weighted echoplanar images $(64 \times 643 \times 3 \mathrm{~mm}$ pixels, TE $=40$ $\mathrm{ms}$ ) sensitive to blood oxygenation level dependent 
(BOLD) contrast. $1.8 \mathrm{~mm}$ thick echoplanar images were acquired axially every $3 \mathrm{~mm}$, positioned to cover the whole brain. Data were recorded during a single acquisition period. A total of 558 volume images were acquired over three sessions (186 volumes per session) with a repetition time (TR) of $4.1 \mathrm{~s} /$ volume. The first six volumes in each session were discarded (leaving 180 volumes per session) to allow for $\mathrm{T} 1$ equilibration effects.

Trials from all conditions were randomly presented in a single-event design. The mean trial time was 12300 ms with a random jitter of $\pm 2050 \mathrm{~ms}$ ( $1 \mathrm{TR})$. Trials duration thus varied from 10.25 to $14.35 \mathrm{~s}$. This variation affected only the times subjects had to respond to the task (3.75-7.85 $\mathrm{s}$ in increments of $68 \mathrm{~ms})$ and not the presentation times of the sentences, which were fixed. Sixty trials were presented during each session for a total of 180 over the three sessions. Each session lasted $12.3 \mathrm{~min}$. The scanner was synchronized with the presentation of all trials in each session.

\subsection{Data analysis}

Data were analyzed using Statistical Parametric Mapping (SPM 99) [6]. All volumes in a session were spatially realigned to the first volume of the session and temporally realigned to the $\mathrm{AC}-\mathrm{PC}$ slice, to account for different sampling times of different slices. Subjects with head movement greater than $3 \mathrm{~mm}$ were discarded. A mean image created from the realigned volumes was coregistered with the structural T1 volume. The structural volumes were then spatially normalized to the Montreal Neurological Institute brain template [5] using nonlinear basis functions [1]. The derived spatial transformation was then applied to the realigned T2* volumes, which were finally spatially smoothed with a $12 \mathrm{~mm}$ FWHM isotropic Gaussian kernel in order to make comparisons across subjects and to permit application of random field theory for corrected statistical inference [25]. The resulting time series across each voxel were high-pass filtered with a cut-off of 120

\begin{tabular}{|c|c|c|}
\hline & Concrete Sentences & Abstract Sentences \\
\hline $\begin{array}{l}\text { Relational } \\
\text { Arguments }\end{array}$ & $\begin{array}{l}\text { Karen is in front of Larry } \\
\text { Larry is in front of Jane } \\
\text { Karen is in front of Jane } \\
\text { Nancy is younger than Karen } \\
\text { Karen is younger than Jake } \\
\text { Nancy is younger than Jake }\end{array}$ & $\begin{array}{l}\mathrm{K} \text { is in front of } \mathrm{L} \\
\mathrm{L} \text { is in front of } \mathrm{J} \\
\mathrm{K} \text { is in front of } \mathrm{J} \\
\mathrm{N} \text { is younger than } \mathrm{K} \\
\mathrm{K} \text { is younger than } \mathrm{J} \\
\mathrm{N} \text { is younger than } \mathrm{J}\end{array}$ \\
\hline Baseline & $\begin{array}{l}\text { Karen is in front of Larry } \\
\text { Larry is in front of Jane } \\
\text { George is above Mary } \\
\text { Nancy is younger than Karen } \\
\text { Karen is younger than Jake } \\
\text { Mary is richer than Jane }\end{array}$ & $\begin{array}{l}K \text { is in front of } L \\
L \text { is in front of } J \\
N \text { is above } J \\
N \text { is younger than } K \\
K \text { is younger than } J \\
K \text { is richer than } J\end{array}$ \\
\hline
\end{tabular}

\section{Event-Related Stimuli Presentation}

312.3 min repetitions 60 event presentations $\mathrm{TR}=4.1 \mathrm{sec}$ Random Jitter

\section{Task: is argument valid?}

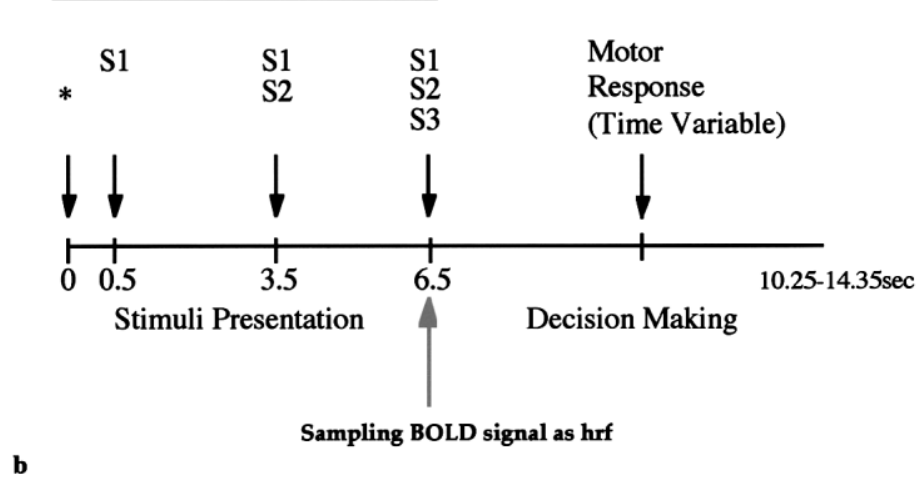

Fig. 1. (a) Sixty concrete and sixty abstract three-term relational arguments were generated. The logically relevant information was identical in both conditions. All sentences were grammatical, meaningful, and of roughly equal length. The conclusions of the arguments were switched around to generate the baseline condition. (b) Stimuli from all conditions were presented randomly in an event-related design. An '*' indicated the start of a trial at $0 \mathrm{~s}$. The sentences appeared on the screen one at a time with the first sentence appearing at $500 \mathrm{~ms}$, the second at $3500 \mathrm{~ms}$, and the last sentence at $6500 \mathrm{~ms}$. The length of trials varied from 10.25-14.35 s, leaving subjects 3.75-7.85 s to respond (after the presentation of the third sentence). 
Table 1

Behavioural scores

\begin{tabular}{llllll}
\hline & RTs (ms) & & & \multicolumn{2}{l}{ Scores (\%) } \\
\cline { 2 - 3 } \cline { 5 - 6 } & Concrete & Abstract & & Concrete & Abstract \\
\hline Reasoning & $3085(776)^{\mathrm{a}}$ & $3283(755)$ & & $78.9(19.4)$ & $75.9(18.1)$ \\
Baseline & $1452(535)$ & $1250(422)$ & & $97.0(5.1)$ & $99.6(1.6)$
\end{tabular}

${ }^{\text {a }}$ S.D. shown in parentheses.

$\mathrm{s}$, using cosine functions to remove section-specific low frequency drifts in the BOLD signal. Global means were normalized by proportional scaling to a grand mean of 100 , and the time series temporally smoothed with a 4 s FWHM Gaussian kernel to swamp small temporal autocorrelations with a known filter.

Condition effects at each voxel were estimated according to the general linear model and regionally specific effects compared using linear contrasts. Each contrast produced a statistical parametric map of the $t$-statistic for each voxel, which was subsequently transformed to a unit normal $Z$-distribution. The activations reported survived a voxel-level correction of $P<0.05$ $(Z>4.60)$ using a repeated measures ANOVA with pooled error term (random effect model). An exception to this correction was made in the case of anatomical symmetry, such that, if a structure in one hemisphere was significantly active, we also reported any corresponding activation in the other hemisphere $(P<$ $0.001)$, even if it did not survive correction. The sampled data were time-locked to onset of the third sentence (i.e. the beginning of the reasoning component of the task), thus avoiding much of the preliminary cognitive activity of reading and processing the premises.

\section{Results}

Behavioral scores indicated that subjects performed the task in the expected manner (see Table 1). Subjects took a mean of $3184 \mathrm{~ms}($ S.D. $=765$ ) (after presentation of the third sentence at $6500 \mathrm{~ms}$ ) to respond to the reasoning task, significantly longer than the $1351 \mathrm{~ms}$ $($ S.D. $=479)$ required to respond to the baseline condition $(t(13)=14.1, P<0.0001)$. Similarly, with a mean of $98 \%$ (S.D. $=0.03)$ correct on baseline trials versus $77 \%$ (S.D. $=0.19$ ) correct on reasoning trials, subjects performed significantly better on baseline trials $(t(13)=6.3, P<0.0001)$. Subjects' response time of $3283 \mathrm{~ms}$ (S.D. $=755)$ for the abstract arguments was significantly longer than their response time of $3085 \mathrm{~ms}$ $($ S.D. $=776)$ for the concrete arguments $(t(13)=3.44$, $P=0.004)$. For baseline trials, response times were longer for concrete trials than abstract trials $(t(13)=$ 3.9, $P=0.002)$. Performance scores for the concrete reasoning condition $(79 \%$, S.D. $=0.19)$ were slightly, but not significantly $(t(13)=1.7, P=0.11)$ better than the scores for the abstract condition $(76 \%$, S.D. $=0.18)$. In baseline trials, performance was slightly better in the abstract condition than the concrete condition $(t(13)=$ $2.1, P=0.059$ ). (We also analysed the behavioural data along the lines of determinate/indeterminate and consistent/inconsistent. Consistent with the published literature, subjects took significantly longer $(3438 \mathrm{~ms}$ $($ S.D. $=590))$ to respond to the inconsistent problems than the consistent problems $(2949 \mathrm{~ms}($ S.D. $=607))$ $(t(13)=6.19, P<0.0001)$. There was, however, no difference in the subjects' scores for consistent $(77 \%$ $($ S.D. $=13.6))$ and inconsistent $(77 \% \quad($ S.D. $=12.4))$ problems $(t(13)=.01, P=0.99)$. Subjects took $3250 \mathrm{~ms}$ $($ S.D. $=560)$ to respond to the determinate problems as compared to $3024 \mathrm{~ms}($ S.D. $=826)$ for the indeterminate problems $(t(13)=1.44, P=0.17)$. Subjects scored $78.8 \%$ (S.D. $=9.8$ ) in the determinate problems compared to $72.5 \%($ S.D. $=23.6)$ in the indeterminate problems $(t(13)=1.41, P=0.18)$.

The fMRI results are summarized in Table 2. The main effect of reasoning, derived from a comparison of concrete and abstract reasoning with the respective baseline conditions as reference ( (abstract reasoning + concrete reasoning $)-$ (abstract baseline + concrete baseline)) revealed activation in bilateral cerebellum, lingual gyrus (BA 17, 18), bilateral middle occipital gyrus (BA 19), bilateral ( $\mathrm{LH}>\mathrm{RH}$ ) superior and inferior parietal lobule (BA 7, 40), bilateral caudate nuclei $(\mathrm{RH}>\mathrm{LH})$, bilateral medial superior frontal lobe (BA 6), bilateral dorsal middle frontal lobe (BA 6), and left dorsolateral frontal lobe (BA 9) (Fig. 2).

The examination of simple main effects revealed that both concrete and abstract reasoning trials actually utilized similar networks, but with differential activation of parietal and occipital regions. A comparison of concrete reasoning with concrete baseline (concrete reasoning - concrete baseline) resulted in activation of bilateral cerebellum, primary visual cortex (BA 17/18), right middle occiptial gyrus (BA 18), bilateral ( $\mathrm{LH}>$ $\mathrm{RH}$ ) inferior and superior parietal lobule (BA 40, 7), precuneus (BA 7) and bilateral $(\mathrm{RH}>\mathrm{LH})$ caudate nuclei. A comparison of abstract reasoning with abstract baseline (abstract reasoning - abstract baseline) resulted in activation of bilateral cerebellum, primary visual cortex (BA 17/18), and bilateral $(\mathrm{RH}>\mathrm{LH})$ superior parietal lobule (BA 7).

A conjunction analysis of concrete and abstract reasoning (conjunction (abstract reasoning-abstract baseline), (concrete reasoning - concrete baseline)) revealed activation in bilateral cerebellum, bilateral lingual gyrus (BA 17, 18), bilateral middle occipital gyrus (BA 19), bilateral superior parietal lobule (BA 7), bilateral caudate nuclei $(\mathrm{RH}>\mathrm{LH})$, bilateral medial superior frontal gyrus (BA 6), bilateral dorsal middle frontal gyrus (BA 6), left middle frontal gyrus (BA 8). 
Table 2

Name and coordinates of brain regions that remained significantly active after each subtraction

Location (Brodmann area) MNI coordinates

\begin{tabular}{lll}
\hline & $Y$ & $Z$
\end{tabular}

Main effect of reasoning

Rt. cerebellum

Rt. cerebellum

Rt. cerebellum

Rt. cerebellum

Lt. cerebellum

Lt. cerebellum

Lt. cerebellum

Calcarine sulcus/lingual gyrus (17/18)

Lt. occipital gyrus (19)

Rt. occipital gyrus (19)

Precuneus (7)

Lt. superior and inferior parietal lobule (40)

Rt. superior and inferior parietal lobule (40)

Rt. caudate nucleus/accumbens

Lt. caudate nucleus/accumbens

Rt. middle frontal gyrus (6)

Lt. middle frontal 1 gyrus (6)

Bi. dorsal medial frontal gyrus (6)

Lt. middle frontal gyrus (9)

Concrete reasoning

Rt. cerebellum

Lt. cerebellum

Rt. calcarine sulcus/lingual gyrus (17/18)

Rt. calcarine sulcus/lingual gyrus (17/18)

Rt. inferior occipital gyrus (18)

Lt. inferior parietal lobule (40)

Rt. inferior parietal lobule (40)

Lt. superior parietal lobule (7)

Rt. superior parietal lobule (7)

Precuneus (7)

Rt. caudate nucleus/accumbens

Lt. caudate nucleus/accumbens

\section{Abstract reasoning}

Rt. cerebellum

Lt. cerebellum

Calcarine sulcus/lingual gyrus (17/18)

Rt. superior parietal lobule (7)

Lt. superior parietal lobule (7)

Conjunction of abstract and abstract concrete reasoning
Lt. cerebellum
Lt. cerebellum
Rt. cerebellum
Rt. cerebellum
Rt. cerebellum
Lt. lingual gyrus (18)
Rt. calcarine sulcus/lingual gyrus (17/18)
Lt. calcarine sulcus/lingual gyrus $(17 / 18)$
Lt. superior occipital gyrus (19)
Rt. superior occipital gyrus (19)
Lt. superior parietal lobule (7)
Rt. superior parietal lobule (7)
Lt. superior parietal lobule (7)
Rt. superior parietal lobule (7)
Precuneus (7)
Rt. caudate nucleus/accumbens
Lt. caudate nucleus/accumbens

((Abstract reasoning + concrete reasoning $)-($ abstract baseline + concrete baseline))

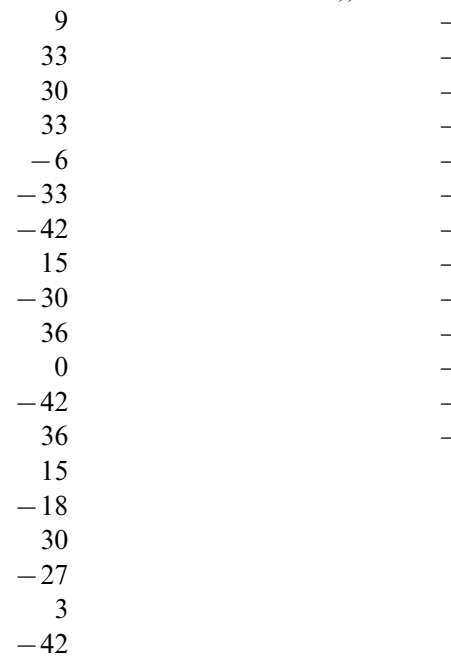

$\begin{array}{rrr}-75 & 27 & 6.08 \\ -66 & -51 & 5.43 \\ -60 & -33 & 4.98 \\ -48 & -45 & 4.87 \\ -75 & -33 & 6.01 \\ -54 & -39 & 5.19 \\ -60 & -33 & 4.86 \\ -99 & 6 & 5.81 \\ -81 & 30 & 4.61 \\ -75 & 33 & * 4.50^{\mathrm{a}} \\ -60 & 48 & 5.69 \\ -51 & 48 & 5.64 \\ -48 & 48 & 5.60 \\ 6 & 0 & 5.14 \\ 0 & -3 & * 4.13 \\ 9 & 51 & 4.64 \\ 3 & 51 & 4.63 \\ 12 & 51 & 4.60 \\ 30 & 33 & 4.63\end{array}$

(Concrete reasoning - concrete baseline)

$\begin{array}{rrrr}33 & -66 & -51 & 4.89 \\ -9 & -75 & -36 & 4.77 \\ 6 & -87 & -3 & 4.66 \\ 9 & -96 & 9 & 5.09 \\ 33 & -87 & 9 & 4.66 \\ -42 & -51 & 48 & 5.14 \\ 36 & -48 & 48 & 3.68 \\ -18 & -66 & 57 & 4.68 \\ 12 & -63 & 57 & * 4.03 \\ 0 & -60 & 48 & 4.64 \\ 15 & 6 & -6 & 4.85 \\ -18 & 0 & -3 & * 3.73\end{array}$

(Abstract reasoning - abstract baseline)

$\begin{array}{rrrr}9 & -78 & -24 & 5.95 \\ -6 & -75 & -33 & 4.82 \\ 18 & -99 & 3 & 5.09 \\ 30 & -60 & 48 & 5.32 \\ -27 & -60 & 51 & * 4.06\end{array}$

(Conjunction (abstract reasoning-abstract baseline) (concrete reasoning-concrete baseline))

$\begin{array}{rrrr}-6 & -75 & -33 & 6.81 \\ -33 & -54 & -39 & 5.84 \\ 30 & -57 & -33 & 5.59 \\ 33 & -63 & -51 & 5.24 \\ 36 & -51 & -51 & 4.95 \\ -12 & -81 & -15 & 5.41 \\ 15 & -99 & 9 & 6.58 \\ -6 & -102 & 0 & 6.53 \\ -30 & -81 & 30 & 5.21 \\ 33 & -72 & 33 & 4.93 \\ -21 & -69 & 51 & 6.19 \\ 24 & -60 & 60 & 5.87 \\ -30 & -60 & 51 & 6.07 \\ 30 & -60 & 54 & * 3.33 \\ 0 & -60 & 48 & 6.23 \\ 12 & 6 & 0 & 5.05 \\ -15 & 3 & -3 & * 4.04\end{array}$


Table 2 (Continued)

Location (Brodmann area)

MNI coordinates

$Z$-score

\begin{tabular}{lll}
\hline$X$ & $Y$ & $Z$
\end{tabular}

Lt. middle frontal gyrus (6)

Rt. middle frontal gyrus (6)

Bi. dorsal medial frontal gyrus (6)

Lt. middle frontal gyrus (8)

Main effect of content

Lt. inferior parietal lobule (40)

Lt. superior parietal lobule (7)

Rt. superior parietal lobule (7)

Rt. inferior occipital gyrus (19)

Lt. inferior occipital gyrus (19)

Main effect of content

Rt. calcarine sulcus/lingual gyrus (17/18)

Lt. calcarine sulcus/lingual gyrus $(17 / 18)$

Lt. calcarine sulcus/lingual gyrus (17/18)

Simple effect of content

Lt. inferior parietal lobule (40)

Rt. superior parietal lobule (7)

Simple effect of content

Rt. calcarine sulcus/lingual gyrus (17/18)

Lt. calcarine sulcus/lingual gyrus (17/18)

Lt. calcarine sulcus/lingual gyrus $(17 / 18)$

$\begin{array}{rrrr}-27 & 3 & 48 & 5.16 \\ 33 & 3 & 51 & 4.64 \\ 3 & 12 & 51 & 5.09 \\ -42 & 30 & 36 & 4.75\end{array}$

(Abstract reasoning + baseline $)-($ Concrete reasoning + baseline $)$

$\begin{array}{llll}-48 & -39 & 51 & 5.62\end{array}$

$\begin{array}{llll}-18 & -66 & 57 & 5.25\end{array}$

$\begin{array}{llll}33 & -54 & 66 & 4.89\end{array}$

$\begin{array}{rrrr}48 & -75 & 0 & 4.78\end{array}$

$\begin{array}{llll}-48 & -75 & -6 & 4.93\end{array}$

(Concrete reasoning + baseline $)-($ abstract reasoning + baseline $)$

$\begin{array}{llll}9 & -84 & 0 & \text { Inf }\end{array}$

$\begin{array}{rrrr}-9 & -81 & 6 & 6.70\end{array}$

$\begin{array}{llll}-6 & -93 & -6 & 6.45\end{array}$

Abstract reasoning-concrete reasoning

$\begin{array}{llll}-45 & -36 & 48 & 4.64\end{array}$

$\begin{array}{llll}27 & -69 & 51 & * 4.30\end{array}$

Concrete reasoning-abstract reasoning

$\begin{array}{rrrr}9 & -84 & 0 & 6.67 \\ -9 & -84 & 6 & 5.07 \\ -12 & -81 & -12 & 4.93\end{array}$

\footnotetext{
a Activations marked with an '*' did not survive correction and are reported only because the corresponding region in the other hemisphere did survive correction.
}

Direct comparisons of concrete and abstract conditions (masked by the main effect of reasoning relative to baseline) confirmed differential activation in parietal and occipital regions. The abstract versus concrete content comparison ((abstract reasoning + baseline) (concrete reasoning + baseline)) revealed significantly greater activation of left superior and inferior parietal lobule (BA 7, 40), right superior parietal lobule (BA 7), and bilateral inferior occipital gyrus (19) in the abstract condition (Fig. 3). The reverse comparison ((concrete reasoning + baseline $)-($ abstract reasoning + baseline $))$ revealed activation only of primary visual cortex (BA 17, 18). The direct simple comparisons of reasoning masked by the main effect of reasoning relative to baseline - revealed similar results. Abstract reasoning compared to concrete reasoning (abstract reasoning concrete reasoning) activated the left inferior (extending to superior) parietal lobule (BA 7, 40), and the right superior parietal lobule (BA 7). As in the main effect of content, activation was also present in the bilateral inferior occipital gyrus (19), but it did not survive correction. The reverse comparison (concrete reasoning - abstract reasoning) resulted in activation of the primary visual cortex (BA 17, 18). There was no interaction between content type and reasoning.

\section{Discussion}

Our results indicate that three-term relational rea- soning implicates a widespread dorsal network incorporating bilateral occipital (BA 17, 18, 19), bilateral parietal (BA 7, 40), bilateral dorsal frontal (BA 6), left dorsolateral prefrontal cortex (BA 9), basal ganglia nuclei and cerebellum regions. This pattern of bilateral occipital, parietal, and frontal activation has been reported in a number of studies involving the manipulation of visuo-spatial information $[2,3,17]$. The primary visual cortex has been activated in imagery tasks $[13,14]$. A bilateral occipital-parietal-frontal network has been reported in studies involving manipulation of allocentric spatial relations (e.g. up, left, front, down, right, and back) such as found in our reasoning task [16]. This network also subserves spatial working memory rehearsal and manipulation processes [12,21-24], and is very similar to that reported for certain types of mathematical reasoning involving approximation of numerical quantities [4]. Picture-sentence verification tasks have been shown to involve greater parietal activation when a visual-spatial strategy is utilized and greater activation of Broca's area when a linguistic strategy is utilized [19]. Thus our profile of activation suggests that during reasoning subjects bypassed the linguistic system and constructed visuo-spatial representations from the sentences, a finding consistent with phenomenological and theoretical claims that subjects solve three-term relational arguments through the use of Venn diagrams, Euler circles, or perhaps the type of spatial models predicted by mental model theory [11]. 
The network revealed by our results encompasses many regions found in previous imaging studies of deductive reasoning $[7-10,18]$, but with some important discrepancies. Some of these discrepancies can be accounted for by methodological and task differences, while others suggest strategy differences in dealing with different types of arguments. For example, a study by Goel et al. [9], using (contentful) three-term relational arguments very similar to the present study, reported left hemisphere language system activation, but failed to report any parietal activation. The Goel et al. [9] study was a $\left[{ }^{15} \mathrm{O}\right] \mathrm{H}_{2} \mathrm{O}$ PET block design in which the activation was averaged over $1 \mathrm{~min}$, much of it being taken up by reading of the argument forms, so it possible that activation associated with the reasoning component of the task was swamped by non-reasoning aspects of the task. Also, the baseline task in the above study involved reading the same argument forms. The parietal system may be very sensitive to explicit spatial relations. If so, simply reading argument forms involving explicit relations may be sufficient to engage it. In this case, any parietal activation associated with reasoning may subtract out. In the present study we controlled for these factors by sampling the BOLD signal after the presentation of the third sentence and used baseline trials that did not constitute arguments (the third sentence being unrelated).

In a related study, using an identical methodology to the present investigation we reported two dissociable networks for concrete (e.g. all apples are red; all red things are fruit; all apples are fruit) and abstract (e.g. all $\mathrm{A}$ are $\mathrm{B}$; all $\mathrm{B}$ are $\mathrm{C}$; all $\mathrm{A}$ are $\mathrm{C}$ ) syllogistic reasoning [7]. In particular, a temporal lobe (BA 21/22) activation was evident in a concrete syllogistic reasoning condition, an activation conspicuously absent in the present study. We found only primary visual cortex activation in the concrete minus abstract comparison. A possible explanation of this is the use of mental imagery in the concrete trials. However, an examination of parameter estimates and comparison of the concrete and abstract baselines suggests that the activation of the striate cortex (BA 17) and lingual gyri (BA 18)

\section{Main Effect of Reasoning}

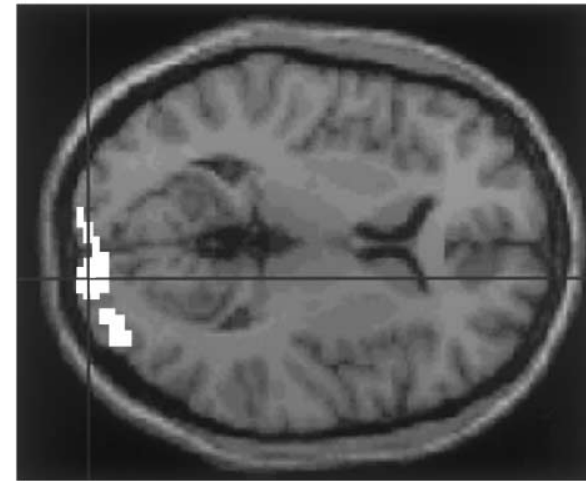

$\mathbf{a}$

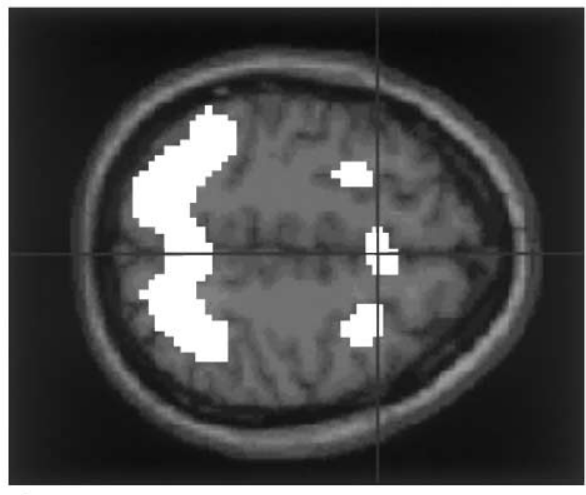

c

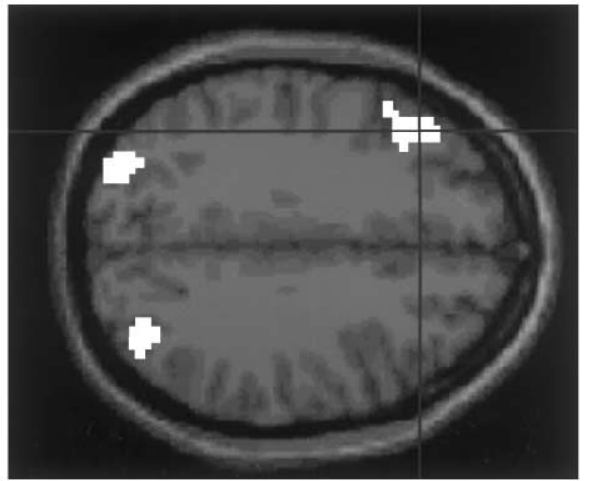

b

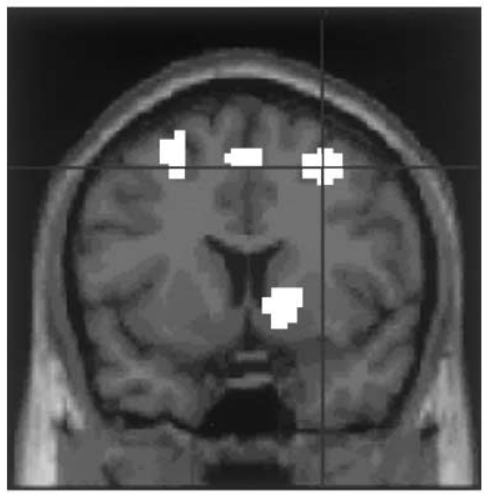

d

Fig. 2. A statistical parametric map (SPM) rendered into standard stereotactic space and superimposed on to transverse (a, b, c) and cronal (d) sections of an magnetic resonance image (MRI) which is itself in standard space. Main effect of reasoning ((abstract reasoning + concrete reasoning) - (abstract baseline + concrete baseline)) activated (a) primary visual cortex $(15,-99,6)$; (b) bilateral occipital gyrus $(-30,-81,30$ and 36, $-75,33)$, left dorso-lateral frontal cortex $(-42,30,33)$; (c) bilateral inferior and superior parietal lobules $(-42,-51,48$ and 36 , -48 , $48)$, precuneus $(0,-60,48)$; (c, d) bilateral middle frontal gyrus $(30,9,51$ and $-27,3,51)$ and dorsal-medial prefrontal gyrus $(3,12,51)$; (d) right caudate nucleus/accumbens $(15,6,0)$; and cerebellum (not shown). All activations survived a voxel-level correction of $P<0.05(Z>4.60)$ using a random effects model. 


\section{Main Effect of Content}
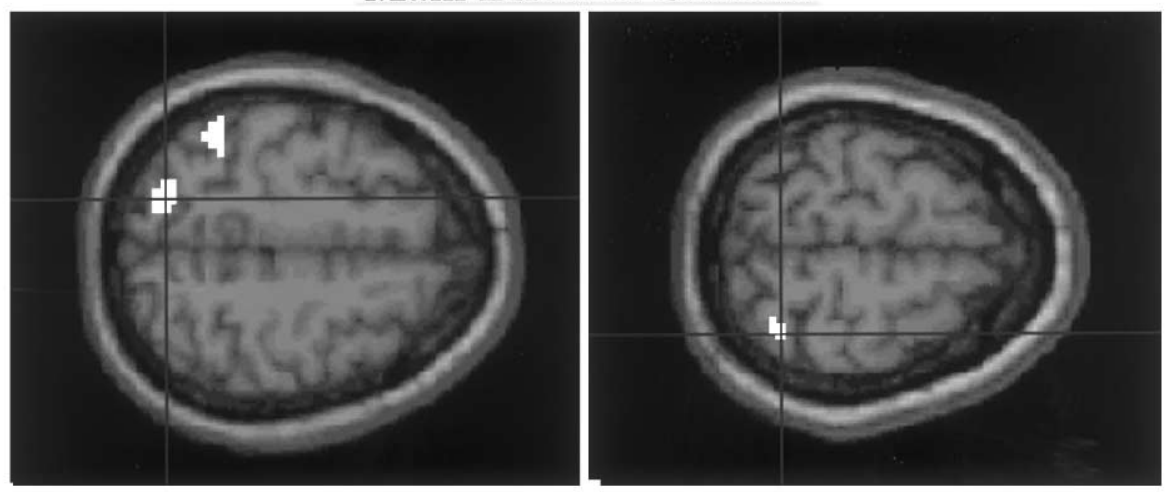

a

b

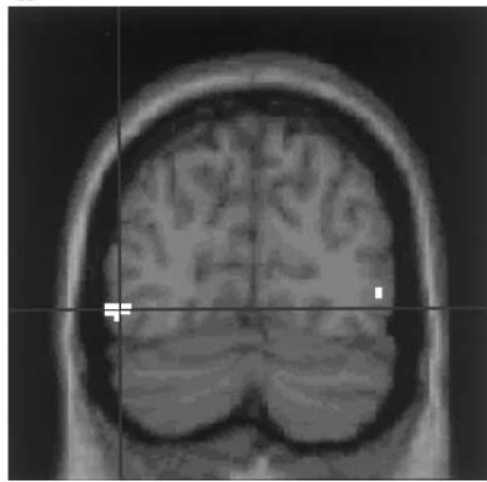

c

Fig. 3. SPMs rendered into standard stereotactic space and superimposed onto transverse $(a, b)$ and cronal (c) sections of an magnetic resonance image (MRI) which is itself in standard space. A direct comparison of the abstract with concrete conditions ((abstract reasoning + baseline) (concrete reasoning + baseline)), revealed greater activation in: (a) left superior and inferior parietal cortex $(-18,-66,57$ and $-48,-39,51)$; (b) right superior parietal lobule $(33,-54,66)$; and (c) bilateral occipital gyrus $(48,-75,0$ and $-48,-75,-6)$. All activations survived a voxel-level correction of $P<0.05(Z>4.60)$ using a random effects model.

reflects greater visual processing due to increased letter lengths of the concrete words (also reported in [7]). For example, 'Mary is behind Paul' has 16 characters and occupies more of the visual field than ' $\mathrm{M}$ is behind P' which has only 10 characters.

The lack of temporal lobe (BA 21/22) activation in the present study might be explained by analysing the nature of the content used in the two studies. The concrete sentences in Goel et al. [7], were syllogisms of the form 'All apples are poisonous' whereas the concrete sentences in the present study are of the form 'John is to the right of Mary'. The former sentence types predicate known properties to known objects. We have beliefs about whether they are true or false. By contrast, the latter sentence types do not allow for such beliefs. (It is possible to generate relational sentences one can have beliefs about, e.g. 'London is north of Rome' or 'granite is harder than diamonds'.) This leaves open the interesting possibility that involvement of BA $21 / 22$ in reasoning may be specific to content processing involving belief networks rather than just concrete contents.

In abstract syllogistic reasoning, Goel et al. [7] reported parietal activation in left hemisphere alone. In the present study, parietal activation was bilateral. The activation of right parietal may be explicable by the presence of explicit spatial relations found in the arguments used in the present study. Another notable difference is involvement of inferior prefrontal cortex in both concrete and abstract conditions of the Goel et al. [7] study, and its absence in the present study. This may suggest genuine processing differences between categorical syllogisms and three-term relational arguments in terms of the involvement of the linguistic system.

While we do not find evidence for two dissociable networks in reasoning about concrete and abstract three-term relational arguments (for the reasons noted above), we do find greater involvement of parietal and occipital lobes in the abstract condition compared to the concrete condition. There are several possible explanations for this. The argument forms in the concrete and abstract conditions were logically matched for difficulty. However, examination of the behavioural scores indicates that subjects took longer to complete the abstract trials and did not do quite as well. One possibility is that the differential parietal and occipital activation in the two conditions reflects differential task difficulty. However, the difference also extends to the 
baseline items, which showed a reverse pattern of behavioural scores, suggesting difficulty may not be the critical factor. The alternative explanation is that parietal lobe activation may reflect a greater sensitivity to spatial relations involving abstract terms.

In conclusion, mental logic theories predict that the language (syntactic) system is both necessary and sufficient for deductive reasoning while mental model theories predict that the visuo-spatial system is necessary and sufficient. For reasoning involving three-term relational arguments, we have found evidence for involvement of a bilateral occipital-parietal-frontal visuo-spatial network as predicted by mental model theories (and introspective phenomonological experience), irrespective of the presence of concrete or abstract sentences. While a similar network is used for both concrete and abstract arguments, there is greater occipital-parietal involvement in the abstract arguments. However, the content utilized in the study was such that subjects could have no prior beliefs about the sentences. It remains to be seen how the presence of arguments containing belief-laden sentences will influence reasoning strategies and mechanisms in three-term relational arguments. Finally, these results are different from those involving syllogistic reasoning [7], suggesting involvement of differential strategies and mechanisms in the two types of reasoning.

\section{Acknowledgements}

VG is supported a McDonnell-Pew Program in Cognitive Neuroscience Award, a National Science and Engineering Council of Canada grant, and a Social Sciences and Humanities Research Council of Canada grant. RJD is supported by the Wellcome Trust.

\section{References}

[1] Ashburner J, Friston KJ. Nonlinear spatial normalization using basis functions. Human Brain Mapping 1999;7:254-66.

[2] Corbetta M, Miezin FM, Dobmeyer S, Shulman GL, Petersen SE. Selective and divided attention during visual discriminations of shape, color, and speed: functional anatomy by positron emission tomography. The Journal of Neuroscience 1991;11:2383-402.

[3] Corbetta M, Miezin FM, Shulman GL, Petersen SE. A PET study of visuospatial attention. The Journal of Neuroscience 1993;13:1202-26.

[4] Dehaene S, Spelke E, Pinel P, Stanescu R, Tsivkin S. Sources of mathematical thinking: behavioral and brain-imaging evidence. Science 1999;284:970-4.
[5] Evans AC, Collins DL, Mills SR, Brown ED, Kelly RL, Peters TM. 3D Statistical neuroanatomical models from 305 MRI volumes. Proc. IEEE-Nuclear Science Symposium and Medical Imaging Conference, 1993:1813-1817.

[6] Friston K, Holmes A, Worsley K, Poline J-B, Frith C, Frackowiak R. Statistical parametric maps in functional imaging: a general approach. Human Brain Mapping 1995;2:189-210.

[7] Goel V, Buchel C, Frith C, Dolan RJ. Dissociation of mechanisms underlying syllogistic reasoning. NeuroImage 2000;12:504-14.

[8] Goel V, Gold B, Kapur S, Houle S. The seats of reason: a localization study of deductive and inductive reasoning using PET (O15) blood flow technique. NeuroReport 1997;8:1305-10.

[9] Goel V, Gold B, Kapur S, Houle S. Neuroanatomical correlates of human reasoning. Journal of Cognitive Neuroscience 1998; 10:293-302.

[10] Houde O, Zago L, Mellet E, et al. Shifting from the perceptual brain to the logical brain: the neural impact of cognitive inhibition training. Journal of Cognitive Neuroscience 2000;12:721-8.

[11] Johnson-Laird PN. Mental models, deductive reasoning, and the brain. In: Gazzaniga MS, editor. The Cognitive Neurosciences. Cambridge, MA: MIT Press, 1994:999-1008.

[12] Jonides J, Schumacher EH, Smith EE, et al. The role of parietal cortex in verbal working memory. The Journal of Neuroscience 1998;18:5026-34.

[13] Kosslyn SM, Pascual-Leone A, Felician O, et al. The role of area 17 in visual imagery: convergent evidence from PET and rTMS. Science 1999;284:167-70 (see comments) (published erratum appeared in Science 1999;284(5416):197).

[14] Kosslyn SM, Thompson WL, Kim IJ, Alpert NM. Topographical representations of mental images in primary visual cortex. Nature 1995;378:496-8.

[15] Laeng B. Lateralization of categorical and coordinate spatial functions: a study of unilateral stroke patients. Journal of Cognitive Neuroscience 1994;6:189-203.

[16] Mellet E, Tzourio N, Crivello F, Joliot M, Denis M, Mazoyer D. Functional anatomy of spatial mental imagery generated from verbal instructions. The Journal of Neuroscience 1996;16:650412.

[17] Nobre AC, Sebestyen GN, Gitelman DR, Mesulam MM, Frackowiak RS, Frith CD. Functional localization of the system for visuospatial attention using positron emission tomography. Brain 1997; 120:515-33.

[18] Osherson D, Perani D, Cappa S, Schnur T, Grassi F, Fazio F. Distinct brain loci in deductive versus probabilistic reasoning. Neuropsychologia 1998;36:369-76.

[19] Reichle ED, Carpenter PA, Just MA. The neural bases of strategy and skill in sentence-picture verification. Cognitive Psychology 2000;40:261-95.

[20] Rips LJ. The Psychology of Proof: Deductive Reasoning in Human Thinking. Cambridge, MA: MIT Press, 1994.

[21] Smith EE, Jonides J. Working memory: a view from neuroimaging. Cognitive Psychology 1997;33:5-42.

[22] Smith EE, Jonides J. Neuroimaging analyses of human working memory. Proceedings of the National Academy of Science USA 1998;95:12061-8.

[23] Smith EE, Jonides J. Storage and executive processes in the frontal lobes. Science 1999;283:1657-61.

[24] Smith EE, Jonides J, Marshuetz C, Koeppe RA. Components of verbal working memory: evidence from neuroimaging. Proceedings of the National Academy of Science USA 1998;95:876-82.

[25] Worsley KJ, Friston KJ. Analysis of fMRI time-series revisitedagain. NeuroImage 1995;2:173-81. 